\title{
De la Représentation Freudienne au Signifiant Lacanien: Sur la Pertinence de L'Interprétation Structurelle de L'Inconscient
}

\author{
Mauricio José d'Escragnolle Cardoso ${ }^{1}$ \\ Universidade Federal do Paraná \\ Rosane Zétola Lustoza \\ Universidade Estadual de Londrina
}

\begin{abstract}
RESUMEN - Cet article voudrait expliciter quelques aspects de l'œuvre freudienne dont Lacan se sert pour la lecture structurale de la psychanalyse. En prenant pour base les indications fournies par le psychanalyste français, il s'agit donc de poursuivre un double but: expliciter les concepts le plus saillants de l'interprétation lacanienne de l'inconscient en montrant que celle-ci est déjà ouverte par l'œuvre de Freud, ainsi que signaler la valeur heuristique de la elaboration de Lacan, dans la mesure où ce parcours contribuerait à rendre plus intelligible certains concepts de la théorie freudienne de la représentation. On conclut par là que la théorie lacanienne ne se réduit nullement à la simple importation et application dans la psychanalyse des concepts provenant de la linguistique structurale.
\end{abstract}

Mots-clés : Freud, Lacan, Représentation, Signifiant, Inconscient

\section{Da Representação Freudiana ao Significante Lacaniano: Acerca da Pertinência da Interpretação Estrutural do Inconsciente}

\begin{abstract}
RESUMO - Este artigo visa explicitar alguns aspectos da obra freudiana dos quais se serve Lacan para a leitura estrutural da psicanálise. Tomando por base as indicações fornecidas pelo psicanalista francês, trata-se de perseguir uma dupla finalidade: explicitar os conceitos mais pregnantes da interpretação lacaniana do inconsciente para mostrar que esta leitura estava já aberta pela obra ela mesma de Freud, assim como assinalar o valor heurístico da elaboração de Lacan, na medida em que tal percurso contribuiria a tornar mais inteligível certos conceitos da teoria freudiana da representação. Conclui-se assim que a teoria lacaniana não se reduz de maneira nenhuma à simples importação não-autorizada e aplicação na psicanálise de conceitos provenientes da linguística estrutural.
\end{abstract}

Palavras-Chave: Freud, Lacan, Representação, Significante, Inconsciente

\section{From Freudian Representation to Lacanian Signifying: The Pertinence of Structural Interpretation of the Unconscious}

\begin{abstract}
Based on the indication provided by Lacan, this paper aims to clarify aspects of Freud's work that provided the structural reading of psychoanalysis. The paper pursues a dual objective: to outline the outstanding concepts of Lacanian interpretation of the unconscious, showing these are the result of an interpretation of Freud's work, and highlight the heuristic value of the development of Lacan, to the extent where this path contributed to make some very difficult concepts of Freud's theory of representation intelligible. It concludes that the Lacanian theory can in no way be reduced to a simple importation and application of the concepts from structural linguistics to psychoanalysis.
\end{abstract}

Keywords: Freud, Lacan, Representation, Signifier, Unconscious

Nous nous sommes peut-être trop habitués à ne voir dans la théorie lacanienne que la simple transposition des concepts linguistiques - provenant de la linguistique saussurienne et de la version lévistraussienne du fonctionnalisme de Jakobson - vers le champ de la psychanalyse. Dans ce sens, la vulgate de l'œuvre de Lacan nous incline à considérer qu'il ne reste plus beaucoup de l'apparat freudien originel, malgré le plaidoyer de la part du psychanalyste français d'une fidèle adhésion doctrinaire aux fondements de l'orientation du maître morave.

1 Endereço para correspondência: Praça Santos Andrade n ${ }^{\circ} 50$, sala 215, ala Alfredo Buffren, Curitiba. Paraná, PR. CEP 80060-240. Fone: (41)3310-2625. E-mail: descragnolle@yahoo.fr
Ce type d'intuition n'est pourtant pas entièrement infondé, car Lacan lui-même a signalé maintes fois au cours de l'évolution de son enseignement qu'il avait été forcé de s'éloigner de la lettre de Freud en fonction des propres impasses produits par la théorie de celui-ci. Selon Lacan, il y avait de l'impensé chez Freud - comme celui par exemple liée au statut du Complexe d'Edipe, à la fonction du Père et donc aux impasses relatifs à la détermination de la fin de l'analyse qui en dérivaient. Il y avait également, selon lui, des limitations immanentes au vocabulaire scientifique freudien, lequel trahirais l'élan fondamental de sa découverte - i.e., un vocabulaire trop marquée d'influences positivistes et organicistes, propre aux modèles épistémologiques en vigueur dans l'univers germanique de la fin du XIXème siècle. 
Cette manière d'aborder la nouveauté apportée par la démarche lacanienne laisse néanmoins à l'ombre ce que, à l'intérieur même de la métapsychologie freudienne, pourrait en autoriser un traitement dit structuraliste. Nous pouvons le dire aussi sous la forme d'une interrogation: qu'est-ce que, dans l'appareil théorique de la métapsychologie freudienne, se laissait entendre, en lui-même, d'un point de vue structural ? Dans quels concepts freudiens Lacan a pu puiser l'orientation dite structuraliste au-delà des apports, déjà si bien connus, provenant d'un Saussure, d'un Jakobson ou d'un Lévi-Strauss? Autrement dit. Il n'y avait rien dans l'œuvre freudienne qui se prêtait, en elle-même, à ce type de traitement?

Nous ne le pensons pas. Effectivement, nous croyons que l'inspiration lacanienne n'a nullement été ni la transposition arbitraire d'une heuristique étrangère à son champ d'application ni le fruit d'une simple contamination par l'euphorie structuraliste d'un modèle unitaire pour une science totale de l'homme.

En prenant pour base les propres indications fournies par Lacan, nous chercherons expliciter les aspects de l'œuvre freudienne qui se sont prêtés au psychanalyste français dans son projet d'une lecture structural de la métapsychologie psychanalytique. Pour cela, nous allons privilégier les éléments constitutifs de la théorie lacanienne du signifiant, dans la mesure où elle constitue le domaine majeur tant du projet de retour à Freud que de la tentative d'une interprétation structural de la psychanalyse.

Au-delà du profit relevant d'une histoire des concepts - dont elle justifierait déjà la présente démarche - nous espérons aussi pouvoir contribuer à l'effort de restitution de l'intelligibilité du texte freudien. En partant des propres indications lacaniennes à propos de ses sources chez Freud, nous visons ainsi jeter un peu de la lumière sur certains concepts de la métapsychologie aujourd'hui encore assez obscurs, comme par exemple celui de représentant-représentation ou ceux relatifs aux aspects langagiers de l'appareil psychique.

Nous pouvons alors, pour en résumer notre intuition de départ, faire nôtre ce que Althusser (1973) avait déjà signalé à propos de la possibilité d'une décalage entre la explicitation d'un concept (à l'intérieur de l'œuvre d'un auteur) et son existence déjà en acte. De même qu'Althusser à propos de Marx, nous considérons que Lacan a essayé de mettre en relief un concept de causalité structurelle chez Freud - implicite tant dans sa conception de la représentation psychique que du fonctionnement inconscient - et qui restait donc non explicité, en retard du point de vue notionnel par rapport à la démarche effective qu'il employait déjà. Une conception implicite alors d'une causalité structurelle dans l'inconscient opérait donc en silence dans la démarche freudienne. En paraphrasant Althusser (1973) sur la pensée marxienne, ce concept était encore "à l'état pratique" (p. 34), donc en ayant besoin d'être explicité, et, peut-être, c'était précisément cela que Lacan a également essayé de faire avec Freud.

\section{Le signifiant et la structure inconsciente}

Commençons alors par évoquer la théorie lacanienne du signifiant. Dès le Discours de Rome, le signifiant est défini par Lacan (1956) en des termes strictement structuralistes, comme un «ensemble d'éléments matériels liés par une structure» (p. 243). En n'existant qu'inscrit dans un réseau, le signifiant est l'élément de base d'une structure synchronique, «en tant que chaque élément y prend son emploi exact d'être différent des autres» (Lacan, 1955, p. 414).

Mais, à la différence de Saussure, Lacan considère que le signifiant n'est pas équivalent à la face signifiante d'un signe linguistique. La définition lacanienne, plus souple que celle de Saussure, comprend alors «depuis le couple d'opposition phonématique jusqu'aux locutions composées» (Lacan, 1953, p. 414), et même davantage. En effet, comme le remarque Soler (2004):

"Peut être dit signifiant, en effet, tout élément discret, isolable et combinable à d'autres éléments également discrets et isolables, susceptible de prendre sens. [...], ça peut être aussi bien une image, voire un geste. Une gifle, par exemple, Lacan l'évoque, peut être un signifiant dès lors qu'elle entre dans une structure combinatoire de représentations, un élément dit somatique également, un type de douleur corporelle, comme on le voit dans les conversions hystériques que Freud a mises à jour" (p. 53).

D'après Lacan, Freud aurait découvert à travers de la névrose une forme particulière de discours, un discours articulé de tout matériel susceptible de servir comme unité de sens : «une névrose consiste en ce qu'au lieu de se servir des mots, le bonhomme se sert de tout ce qui est à sa disposition» (Lacan, 1955/1956, p. 285).

En fait, malgré la souplesse de la détermination de la forme du signifiant, nous comprenons que cette conception ne peut être validée sans le recours à une théorie structuraliste. Or, c'est-elle qui permet de penser le signifiant comme un élément discret et différentiel. Ainsi, moins que une langue proprement dite, ce qui importe pour la définition minimale du signifiant, c'est la définition d'un «système synchronique des couplages différentiels" (Lacan, 1957/1966, p. 501) dont la nature de l'élément demeure indifférente. Autrement dit, pour pouvoir définir l'essence des unités signifiantes, il suffit de postuler qu'elles sont soumises «à la double condition de se réduire à des éléments différentiels derniers et de les composer selon les lois d'un ordre fermé» (Lacan, 1957/1966, p. 501).

Dans la recherche d'un modèle minimal pour cette unité, Lacan propose le concept de lettre comme désignant l'essence matérielle du signifiant. À travers lui, Lacan cherche à souligner la différence de son signifiant psychanalytique par rapport au signifiant linguistique. Pourtant, à l'instar de ce qui se produit pour le signifiant linguistique, l'unité de la lettre repose sur les coupures qui caractérisent le langage et implique de considérer l'ordre symbolique en tant qu'ordre segmentaire. Ainsi, du simple fait que le signifiant soit un segment, découlent deux caractéristiques essentielles de celui-ci : premièrement, il désigne un élément différentiel - chaque signifiant est différent de toutes les autres segmentations. Et, deuxièmement, que la valeur particulière d'un segment dépend de la place qu'il occupe par rapport aux autres au sein d'une structure.

Une structure ainsi définie exclue toute théorie nominaliste ou représentationaliste du langage et donc toute référence à des objets extralinguistiques. Un signifiant n'est donc 
pas quelque chose de l'ordre de l' «indice naturel" (Lacan, 1957, p. 418). Comme souligne Lacan (1955/1956): “Le système du langage, à quelque point que vous le saisissiez, n'aboutit jamais à un index directement dirigé sur un point de la réalité, c'est toute la réalité qui est recouverte par l'ensemble du réseau du langage" (p. 61).

Cette subsumption de la réalité par le langage implique «l'absolue non-équivalence du discours avec aucune indication. Si réduit que vous supposiez l'élément dernier du discours. Jamais vous n'y pourrez vous y substituer, ni substituer simplement l'index" (Lacan, 1955/1956, p. 61). C'est cette perspective de la subsomption complète de la réalité par l'ordre symbolique qui rend possible le projet d'une linguistique généralisée, puisant ses racines dans l'œuvre lévisstraussienne. Mais pour comprendre la spécificité du concept de signifiant dans la psychanalyse, il est encore nécessaire d'approfondir la référence à l'œuvre freudienne.

\section{Les racines freudiennes du concept de signifiant (I): les signes de perception}

Lors de la séance du 21 mars 1956 de son troisième séminaire Les Psychoses, Lacan (1955/1956) met 1'accent sur deux moments de l'élaboration freudienne, fondateurs dans sa conception du signifiant. Le premier, Lacan le trouve dans la Lettre 46, lorsque Freud (1896a) affirme que le psychisme fonctionne, entre la mémoire et le discours, comme un système de traduction. Par rapport à cette lettre, Lacan souligne l'importance du fait que, entre les traits mnésiques d'une expérience passée et les représentation-mot (relatives à la conscience), intervient un processus de traduction. Mais, l'importance de cette conception freudienne ne se réduit pas au fait de la traduction. Elle est due aussi à l'hypothèse de la présence d'un reste intraduit, reste qui concerne «l'excédent sexuel» (Freud, 1896a, p. 145), dont Lacan ne se prive pas de signaler l'importance.

Le second moment est marqué, lui, par la Lettre 52, dans laquelle Freud (1896b) poursuit son travail de détermination des strates de l'appareil psychique. Freud y expose alors un appareil psychique organisé en des diverses couches, suivant une stratification qui va de l'inscription de simples traits simultanés de perception jusqu'aux représentations qui forment la conscience. Freud nous dit ainsi que:

\footnotetext{
"Notre mécanisme psychique s'est établit par un processus de stratification: les matériaux présents sous forme de traces mnémoniques se trouvent de temps en temps remaniés suivant les circonstances nouvelles. Ce qu'il y a d'essentiellement neuf dans ma théorie, c'est l'idée que la mémoire est présente non pas une seule mais plusieurs fois et qu'elle se compose de diverses sortes de signes" (p. 153).
}

Pourquoi Lacan considère-t-il que ces deux moments de l'élaboration freudienne sont essentiels dans l'élaboration de son propre concept de signifiant?

Dans la Lettre 46 (Freud, 1896a), les signes de perception (Wahrnehmungszeichen) sont déjà de l'ordre des traits mnésiques, ce sont, autrement dit, des traits appartenant à la mémoire. Ce serait à partir de la retranscription des traits mnésiques (découlant de la perception) dans le système préconscient/conscient sous la forme de représentations-mot que l'activité consciente deviendrait possible. La retranscription est ici indissociable, nous affirme Freud, d'une traduction des éléments d'un système appartenant à un régime symbolique vers un autre système régi par une organisation logique différente. Lacan relève la distinction freudienne entre les différentes couches, c'est-à-dire le caractère stratifié de l'organisation de l'appareil psychique. Ces strates correspondent à maintes et différentes formes de traits et d'organisation logique de ces traits. Fidèle à Freud, Lacan considère que la première couche de traits mnésiques est celle de la perception dans son ensemble synchronique. Pour Lacan, cette couche ne se réduit point à la perception d'un ensemble d'objets donnés de manière simultanée dans un même champ sensible. L'essence de la perception n'est donc pas une gestalt sensible ni même une réalité pré-symbolique, mais la constitution d'un système signifiant.

“L'étape warhnehmung qui est la véritable étape primaire
purement hypothétique, elle est là pour marquer qu'il faut sup-
poser quelque chose de simple à l'origine de ce dont il s'agit,
c'est-à-dire de cette conception de la mémoire comme étant
essentiellement faite d'une pluralité de registres. La première,
c'est donc la première registration des perceptions tout à fait
inaccessibles à la conscience elle aussi, et qui est ordonnée par
des associations de simultanéité. [...] La naissance du signi-
fiant, c'est la simultanéité, et aussi bien l'existence du signifiant
est une coexistence synchronique" (Lacan, 1955/1956, p. 333).

Lacan associe donc cet ordre synchronique des traits mnésiques au registre du signifiant. Ainsi, le refoulement, par exemple, devrait-il être lu comme le refus de la traduction/ transcription d'un ensemble de traits mnésiques d'un système à un autre. Dans ce cas, «la traduction en signes de la nouvelle phase semble être gênée» (Freud, 1896b, p. 157). Toujours selon Lacan (1955/1956):

\begin{abstract}
"Le signifiant de ce qui est enregistré à une de ces étapes en passant à une autre, ne franchit pas le mode de reclassement après-coup que nécessite une phase nouvelle d'organisation signifiant-signification où entre le sujet, et que c'est comme tel et ainsi qu'il faut expliquer l'existence de quelque chose qui est refoulé. Cela reste dans un mode d'inscription qui est antérieur; la notion d'inscription, d'insertion de tout ce qui est dans un signifiant, qui lui-même domine tout, qui domine l'enregistrement, est essentielle à la théorie de la mémoire, pour autant qu'elle est à la base, pour Freud, de sa première investigation du phénomène de l'inconscient" (p. 334).
\end{abstract}

La référence aux signes de perception revient dans son texte sur D'une question préliminaire à tout traitement possible de la psychose (Lacan, 1958, p. 558). A cette date, Lacan vise à reprendre le système d'inscription/retranscription afin de mettre en exergue la différence entre la mémoire de traits synchroniques, et le refoulement, comme interdiction de la retranscription d'un système à l'autre et ce pour souligner que l'inconscient n'est pas la mémoire mais le travail de sélection des traits ainsi que la logique de traduction. S'il s'agit de mémoire, la référence lacanienne ne peut être chez 
Freud que les signes provenant de la perception, donc aux Wahrnehmungszeichen.

C'est dans ce sens que Lacan (1964/1965) fait référence au caractère signifiant des Wahrnehmungszeichen:

"[...] dans le premier schéma, celui qu'il nous donne dans la lettre 52, il [Freud] nous dit qu'il doit y avoir un temps, une étape, où ces Wahrnehmungszeichen, auxquelles il y a lieu de donner tout de suite, d'après ce que je vous ai enseigné, leur vrai nom, à savoir des signifiants [...]Qu'est-ce que c'est, si ce n'est la synchronie signifiante?" (p. 54-55).

C'est donc, d'une part, du fait du caractère d'ensemble synchronique que les Wahrnehmungszeichen sont des signifiants, tandis que, de l'autre, ce type de Zeichen permet de considérer l'inconscient comme une forme vide, en tant qu'un registre formel de traduction établi entre différentes strates du psychisme.

\section{Les racines freudiennes du concept de signifiant (II): le représentant-représentation}

Lacan procède aussi à l'affirmation de l'équivalence entre le signifiant et le représentant-représentation, c'est-à-dire le concept freudien de Vorstellungsrepräsentanz. Le représentant-représentation (Vorstellungsrepräsentanz) désigne toute manifestation de la pulsion dans le psychisme. Il désigne également l'unité matérielle de l'inconscient en tant que complexe associatif de traits investis par la libido. Cette unité du trait d'inscription et de la libido peut être disjointe dans ces composants (ce qui est évident dans le refoulement): d'une part, la représentation proprement dite, la Vorstellung, et de l'autre, le quantum pulsionel, soit-il compris du point de vue de l'affect soit de la libido.

Dans son séminaire Éthique de la Psychanalyse, Lacan (1959/1960), en parlant de l'Esquisse d'une psychologie scientifique de Freud (1895), considère que l'espace existant entre la perception et la conscience est l'espace propre aux Vorstellungsrepräsentanz. C'est dans cette place intermède qu'il se situe ce qui ne relève plus de l'empirisme de la perception ni pourtant de l'ordre des processus secondaires du registre préconscient/conscient, mais d'un ensemble de traits mnésiques qui fonctionnent comme des complexes symboliques. C'est alors dans cette région psychique, celle des processus primaire que nous retrouvons la même structure que le signifiant.

“Ce qui n'est pas simplement Vorstellung, mais comme Freud l'écrit, plus tard, dans son article sur le Unbewußt, Vorstellungsrepräsentanz, ce qui fait de la Vorstellung un élément associatif, un élément combinatoire, qui en fait quelque chose qui, d'ores et déjà, met à notre disposition un monde de la Vorstellung déjà organisé selon les possibilités du signifiant comme tel, quelque chose qui, déjà au niveau de l'inconscient, s'organise selon des lois qui, Freud l'a bien dit, ne sont pas forcément les lois de la contradiction, les lois de la grammaire, mais qui sont d'ores et déjà les lois de la condensation, les lois du déplacement, celles que j'appelle pour vous les lois de la métaphore, les lois de la métonymie» (Lacan, 1959/1960, p. 101)
Pour offrir une image de la structure des représentant-représentations (Vorstellungsrepräsentanz) dans l'inconscient, nous pouvons nous référer au modèle que Freud à élabore pour rendre compte de l'expérience primordiale de satisfaction et de la répétition qui en découle. Lorsque le bébé fit l'expérience de la satisfaction susceptible de faire cesser l'état d'urgence (corrélatif à l'augmentation de l'excitation psychique), les représentations ou traits mnésiques associés à cette expérience demeurent enregistrés dans la mémoire. La fois suivante, où l'état d'urgence se manifeste à nouveau, la tendance de l'appareil est au réinvestissement du complexe associatif qui constitue le souvenir de l'expérience de satisfaction. Cette forme de satisfaction hallucinatoire n'est cependant pas capable de produire la cessation de l'état d'urgence. Le souvenir doit donc être remplacé par une activité perceptive en mesure d'indiquer la présence effective de l'objet.

Il apparaît donc nécessaire que l'appareil psychique soit capable d'établir la distinction entre une représentation-souvenir et une représentation-perception. L'appareil cherche toujours une perception qui soit équivalente au souvenir, dont la conséquence est la définition de l'objet comme objet retrouvé, c'est-à-dire en tant qu'objet soumis à la répétition. Néanmoins, selon Freud un souvenir e une perception peuvent difficilement coïncider complètement. Ainsi, en fonction de l'intersection entre souvenir et perception actuelle, Il restera toujours entre les deux une discordance irréductible. Dans ce sens, à cote de ce que se répète en tant qu'objet familier de l'expérience de satisfaction, subsiste toujours un noyau d'étrangéité irreprésentable et qui échappe à la compréhension.

Nous en avons comme conséquence que l'activité de comparaison entre les complexes perceptif et mnésique met en évidence qu'un complexe quelconque est perçu par le psychisme comme constitué de deux parties distinctes : l'une, une partie constante et incomprise que Freud appelle la Chose (Das Ding) et, l'autre, une composante variable et compréhensible. Le représentant-représentation est ce complexe lui-même formé par un ensemble de traits capables d'inscription symbolique plus un reste incompris, un excédent qui, en tant que tel, est une pure quantité d'excitation investie sur le trait (et que Freud désignait sous le vocable de fixation). Ainsi, le représentation-représentation est-il ce trait mnésique (ou complexe de traits) qui comporte comme un composant inhérent à soi-même, c'est-à-dire du fait même d'être un trait, une portion constante qui échappe à la représentation.

Les signifiants gravitent ainsi autour de quelque chose d'irreprésentable qui doit être compris comme étant l'élément hétérogène au signifiant. Cet élément est pourtant un corrélat direct du signifiant, c'est-à-dire un sous-produit non-symbolisable du procès lui-même de symbolisation. Autrement dit, ce reste étrange qui résiste à la symbolisation des Vorstellungen est une propriété intrinsèque au système de représentation, et d'aucune manière une réalité extra-symbolique.

"[...] ces Vorstellungen, il [Freud] nous affirme que leur gravitation, leur mode d'échange, leur économie, la façon dont elles se modulent, c'est, et il l'articule, selon les mêmes lois où nous 
pouvons reconnaître celles qui, si vous suivez mon enseignement, sont les lois les plus fondamentales du fonctionnement de la chaîne signifiante" (Lacan, 1959/1960, p. 102)

Nous retrouvons ainsi au sein même de cette économie symbolique, dans ce système d'échanges que est la chaîne signifiante, ce caractère du trait d'être toujours élément d'un ensemble. Du fait structural d'être caractérisé fondamentalement comme élément discret, chaque élément signifiant se détermine uniquement dans sa relation différentielle aux autres éléments du système dont il fait partie. Mais, et là nous touchons au cœur de la définition même de système symbolique, cet aspect différentiel du signifiant impliquerait la propriété de ne pas être identique à soi-même, et donc en ayant toujours besoin de son renvoi à l'ensemble dont il fait partie. Autrement dit, chaque trait signifiant porterait ainsi toujours, incluse dans sa propre détermination, un résidu d'indétermination, cet élément autour duquel tournent toutes les Vorstellungen. Ce lien intime d'entre la détermination et l'indéterminé, caractéristique fondamental de tout ensemble symbolique, se donne à considérer comme le principe même de la structure et du système qui la matérialise. Propriété dont Lacan avait déjà observée comme intrinsèque aux Vorstellungsrepräsentanz:

«Vorstellungsrepräsentanz, ce qui, dans l'inconscient, représente comme signe la représentation comme fonction d'appréhension, donc que se représente toute représentation pour autant qu'elle évoque le bien que das Ding apporte avec lui» (Lacan, 1959/1960, p. 116).

Le Vorstellungsrepräsentanz est ainsi le signifiant en tant que tel, autrement dit indissociable du quota d'indétermination qu'indique la Chose. Comme la Chose et le sujet ont en commun une même propriété de ne pas être réduites au signifiant, ils peuvent être mis en équivalence fonctionnelle. A notre avis, Lacan fait ainsi mention de cette caractéristique du structuralisme psychanalytique qui consiste à concevoir le système de signifiants comme ayant parmi ses éléments le sujet lui-même, en tant que forme vide qui possède son homologue dans la Chose.

Comment nous devons alors comprendre la conception lacanienne de la structure du langage pour qu'elle soit compatible avec l'hypothèse freudienne de l'inconscient, c'est-àdire en tant que système de Vorstellungen dans son rapport à Das Ding ? Pour cela, nous devons d'abord nous débarrasser de la représentation habituelle selon laquelle le langage serait un instrument au service d'un sujet transparent à soi-même et dont la fonction est la nomination des choses données empiriquement aux sens. Le langage n'est pas défini, selon Lacan, par sa capacité de désigner un référent sensible. Cette perspective réaliste nous donnerait de surcroit l'idée que c'est la réalité extra-symbolique une sorte de limite externe qu'en fournirait un fondement naturel à l'ensemble du langage.

Au contraire, Lacan cherche la possibilité de lire Freud et sa théorie des représentations à partir d'un point de vue non-référentiel du langage, plutôt proche d'une conception formaliste de la poésie. A la suite de la phonologie jakobsonienne, Lacan vise interpréter la notion freudienne de représentant-représentation à partir d'une espèce de théorie des signifiants-métaphores afin d'expliciter pourquoi il serait en fait impossible pour le sujet d'énoncer une signification d'objet qui soit adéquat au désir.

Toujours dans une espèce d'emploi métaphorique, chaque représentation-signifiante serait quelque chose qui viendrait à la place d'un autre dont le statut serait d'être, au même temps, original et perdu. Ces signifiants, compris alors en tant que métaphore, tiendraient ainsi ce statut quelque peu spécial, d'être toujours dans une occurrence impropre - parce en venant comme substitut d'un autre terme, dont il serait la métaphore, justement - et, propre, parce que cet élément original n'a jamais en fait existé. C'est dans ce sens que nous pouvons comprendre pourquoi la signification pleine du désir, en étant de l'ordre de l'impossible, amène Lacan alors à postuler que le système des représentations-signifiants doit être autoréférentiel.

Nous y avons la raison par laquelle le signifiant ne constitue point de signe et donc la caractéristique est d'être toujours séparé du signifié. Dans des occurrences, par définition, métaphoriques, la seule propriété alors effectivement pertinente d'une représentation-signifiant est celle d'être uniquement différentielle.

Nous voyons ainsi que cette caractéristique différentielle du signifiant ne doit pas être entendue au sens d'une opposition entre des termes contraires. Autrement dit, le rapport entre deux signifiants ou entre deux représentations n'est pas celle de la contrariété, car celle-ci, en étant une différence attributive, c'est-à-dire prédicative, pose en fait qu'il s'agit d'un rapport entre des signifiés. Dans l'opposition - ou dans une relation de contrariété, ce qui en revient au même - par exemple, entre le Chaud et le Froid, nous avons toujours deux qualités ou deux substances qualitativement différentes qui en constituent les deux pôles mutuellement excluents d'un même continu. En plus, en étant les deux extrêmes d'un seul axe qualitatif, ils doivent en fait participer d'une même classe attributive qui les recouvre - dans notre exemple, la température. Il s'agit, nous y pouvons alors voir, d'une différence de type taxonomique, dont l'exemple majeure est la différence aristotélicienne entre des espèces qui pourtant se regroupent dans un même genre. Finalement, dans cette relation de contrariété, nous avons alors toujours la présupposition d'une même classe prédicative, d'un horizon commun qu'oriente l'axe de l'opposition. Ce n'est pas ce type de différence qui Lacan cherche à comprendre et à partir duquel il vise la relecture de la théorie freudienne des représentations inconscientes. En citant Lacan (1955/1956):

"Le jour vient à la présence du jour et sur un fond qui n'est pas un fond de nuit concrète, mais d'absence possible de jour, où la nuit se loge, et inversement d'ailleurs, le jour et la nuit sont là très tôt comme signifiants et non pas comme alternance de l'expérience» (p. 274).

Si dans une relation d'opposition entre contraires, le Jour s'oppose à la Nuit ; alors, dans un rapport de pure différence, non-qualitative, en revanche, le jour ne peut s'opposer en fait qu'à sa propre absence. Il s'agit, dans ce cas de figure, d'une forme de négation non-attributive, et donc plus fondamentale : la négation d'un terme du système n'implique pas son 
contraire prédicatif, mas justement une espèce de fondement absent, c'est-à-dire simplement d'un vide.

Remarquons que, dans les deux types de systèmes - celui formé par les seules différences et celui constitué par des oppositions prédicatives - nous avons toujours un rapport entre au minimum trois éléments. Dans le système d'oppositions qualitatives, les trois termes sont, d'abord, les deux termes opposés, c'est-à-dire les espèces, puis le genre ou classe, dont ils font partie. Il s'agit alors bien de trois éléments, mais répartis dans deux ordres hiérarchiquement distincts. En revanche, dans le système purement différentiel, les trois termes appartiennent au même registre et donc il s'agit, dans ce cas, d'une espèce de classe qui ne constitue pas un sousensemble d'aucun autre. Ainsi, tandis que dans le système de différences oppositives-attributives, nous sommes forcés de considérer un plan hiérarchiquement supérieur qu'en constitue le métalangage du pair d'opposés. Dans le système de pures différences, il n'y a pas la différence entre des niveaux distincts de langage, mais uniquement un rapport différentiel entre les éléments tous de même niveau. Plus précisément, dans ce cas, il s'agit alors d'une différence entre deux éléments signifiants-représentationnels et un troisième élément radicalement hétérogène : le vide lui-même.

La conséquence théorique de la distinction entre ces deux types de différences - disons-nous plus proprement, entre un système basé sur l'opposition et l'autre sur la différence est celle entre un système qui présuppose l'opposition entre langage-objet et métalangage et un autre système pour lequel il n'y a pas de métalangage. En fait, dans ce dernier cas, il s'agit d'un système qui ne s'oppose à plus rien, c'est-àdire, qui ne connaît pas l'opposition. Le corrélat direct d'un système qui ne constitue pas un langage-objet pour un autre métalangage est justement le surgissement d'un ensemble autoréférentiel et donc responsable nécessairement par l'apparition des paradoxes de l'autoréflexion ${ }^{2}$. Il est important alors de signaler que cette absence d'opposition et donc de

2 A propos des rapports entre la distinction des niveaux de métalangage, les ensembles autoréférentiels et le surgissement des paradoxes de l'autoréflexion. Nous voudrions uniquement souligner que la Théorie Simples des Types de Russell est justement une théorie du métalangage logique dont le but est d'empêcher le surgissement des paradoxes de l'autoréférence. S'extraire des impasses de l'autoréférence, passe selon Russel, par le recours au Principe du cercle vicieux qui constitue, en fait, sa Théorie Simple des Types. Grosso modo, la théorie des types refuse l'existence d'ensembles qui puissent contenir eux-mêmes comme élément, en d'autres termes, qu'une totalité puisse être membre d'elle-même : sa loi fondamentale est celle qu'affirme qu'un objet ne peut être défini en tant qu'un ensemble dont il fait partie. La conséquence de la théorie des types, c'est l'avènement d'une nouvelle division de types d'ensemble : d'un côté, ceux qui forment une totalité consistante - à condition d'être déterminés par un concept de niveau supérieur -, et, de l'autre, des multiplicités non-totalisables ou ensemble inconsistants, dont la caractéristique est d'impliquer les paradoxes de l'autoréférence. Afin d'empêcher donc le surgissement d'antinomies, la théorie de types, une théorie métalinguistique, nous interdit ainsi de totaliser certains types de multiplicités, ou encore de former des totalités inconsistantes, sous peine de générer des paradoxes d'auto-inclusion. De cette manière, selon la théorie des types, chaque proposition doit être déterminée comme appartenant à un niveau supérieur au type d'objet sur lequel elle porte, c'est-à-dire d'être basé dans l'opposition entre langage-objet et métalangage. Sur ce sujet, voir Russel, Bertrand. Écrits de logique philosophique. Paris: P.U.F., 1989. Et, également, Koyré, Alexandre. Épiménide - le menteur. Paris: Hermann et compagnie, 1947. distinction de niveau entre langage-objet et métalangage a été également signalé par Freud (1900/2004) dans L'interprétation des rêves:

"Tout se passe comme si, dans une équation algébrique contenant, à côté des chiffres et des lettres, un signe +ou -, ou encore un signe de puissance ou de racine, quelqu'un prenait pour des chiffres les signes d'opérations et mettait dans le désordre les signes des deux catégories». (p. 385)

En ne reconnaissant pas la distinction de niveau entre des éléments et des opérations sur des éléments, donc en s'assoyant sur l'inexistence du métalangage, le système des signifiants-représentations inconscientes devient inconsistant, c'es-à-dire se construit autour d'une contradiction fondamental ou d'une impasse concernant la symbolisation. Si le système ne permet pas alors une exception à-soi, caractéristique propre des ensembles normaux non-contradictoires, l'ensemble des représentations devient ce que Lacan appelle «pas-tout», c'es-à-dire centré sur la contradiction. Ce n'est pas autre chose qu'en dit Freud lorsqu'il signale le fait que l'inconscient ignore le principe de contradiction.

Ainsi, en tant que réseau, le signifiant n'existe qu'au pluriel comme sténographe le concept de chaîne non-hiérarchique de représentations. Plutôt que de désigner l'idée d'une série linéaire des signifiants, le concept de chaîne fait cependant référence à une duplicité minimale nécessaire à la constitution du signifiant : il n'y a du signifiant que dans un rapport avec un autre signifiant. Néanmoins, il faut se garder ici de comprendre l'absence des différents niveaux de métalangage à propos du système de représentations inconscients, comme l'équivalent de l'affirmation qu'il n'y aurait pas d'autre système psychique que celui-là. Au contraire, Freud également observe la nécessité de postuler un deuxième système de représentations-signifiants qu'en fonctionnerait autrement que l'inconscient. La nouveauté freudienne à ce propos, c'est de postuler que ces deux systèmes ou ensembles de représentations sont simplement parallèles, c'est-à-dire qu'ils ne forment pas un couple langage-objet/métalangage.

\section{Fécondité de la théorie lacanienne du signifiant}

Au but de valider la fécondité de la théorie lacanienne du signifiant, nous pouvons la tester sur le modèle de la duplicité des chaînes du texte de Freud (1983) sur les Aphasies. Voyons cet exemple de plus près.

Freud - à l'inverse de la théorie des localisations (de Broca, Lichtheim et Wernicke) qui postule une origine anatomique aux troubles aphasiques du langage - considère que les aphasies sont des troubles fonctionnels relatifs à l'appareil de langage. Cette conception annonce déjà les constructions de 1'Esquisse (Freud, 1895) en proposant un appareil structuré à la base d'un trouble du langage. Contrairement aux thèses de la théorie des localisations, celle des centres corticaux et de l'élémentarisme des représentations, Freud propose celle du procès (l'élément est déjà une association) et de l'appareil (une totalité structuré).

Ainsi qu'à l'encontre l'explication mécanique des fonctions symboliques, Freud (1983) proposait d'établir un 
parallélisme psychophysiologique dans lequel le substrat organique n'est pas la cause du psychique, mais son support. La conception freudienne, déjà à l'époque, est si peu épiphénoménale à l'égard des fonctions langagières que l'appareil n'a même pas une origine innée. L'appareil de langage n'est pas présent lors de la naissance de l'individu, il se développe progressivement dans la relation à un autre appareil de langage déjà, lui, constitué.

Dans ce texte sur les Aphasies, Freud nous fournit un schéma des rapports entre la représentation inconsciente et la représentation de mot ou, plus précisément, entre l'association d'objet et la représentation-mot. Ainsi, Freud (1983), dans le chapitre VI des Aphasies, rappelle que «pour la psychologie, le 'mot' est l'unité de base de la fonction de langage, qui s'avère être une représentation complexe, composée d'éléments acoustiques, visuels et kinesthésiques» (p. 123)

Dans ce schéma, les deux systèmes (des représentations-mot et des associations d'objet) sont caractérisés par la présence d'une association complexe d'images ou traits différentiels (non-représentatifs). Remarquons ainsi que nous ne nous inscrivons plus dans le domaine des impressions sensibles mais dans celui de l'ordre symbolique : dans les deux systèmes, il s'agit toujours d'un ensemble de traits dans une association, formant une invariance dans un rapport à un autre système. Mais, alors, quels sont les critères qui nous permettent de distinguer une représentation-mot d'une association d'objet?

Alors que le schéma de la représentation-mot présente un caractère fermé dans son complexe associatif, les associations d'objet, elles, décrivent un complexe associatif ouvert. De quoi est-il question dans cette distinction entre les caractères ouvert et fermé des deux types de complexe d'associations? Nous considérons que l'association entre les deux complexes constitue le modèle freudien de la semiosis métapsychologique, au sein duquel le lien à l'association d'objet confère une signification au mot. Mais, nous savons déjà que les associations d'objet ne peuvent pas être un référent. Elles ne sont pas simplement l'impression passive d'une chose, mais un autre réseau, aussi symbolique que tout autre, et qui s'associe au premier. La semiosis, dans ce cas, est la transposition d'un terme symbolique provenant d'un système - par exemple, une représentation-mot et le registre préconscient/ conscient - vers un autre registre de traitement, que change la nature de l'élément ainsi que la logique de son fonctionnement - l'association d'objet et l'inconscient.

L'essentiel, ici, c'est que Freud semble suggérer que le domaine des représentations-objet subsume et incorpore les représentations-mot, contrairement à ce que nous serions tentés de penser. Autrement dit, les représentations-mot ne peuvent constituer leur système qu'à partir d'une exclusion. C'est pour cette raison qu'ils forment un système fermé, un système qui se limite par rapport à une extériorité. En revanche, le système de représentations-chose ne s'oppose à rien, il implique une subsomption généralisée et, ainsi, forme un réseau ouvert. Du système d'associations d'objet à celui de représentations-mot, nous passons d'un ordre de différences (qui ne connaît donc pas l'extériorité comme un dehors de soi-même) à celui d'oppositions dualistes.

Le rapport entre les caractères fermé et ouvert est ainsi clarifié: il s'agit de la relation entre un ensemble ouvert sans extériorité, caractéristique des associations d'objet, dont l'ouverture est cette partie constante et incomprise qui forme la Chose; et le registre des représentations-mot, qui existe à partir du moment où il a été expulsé au moins un élément (comme tout ensemble normal). Le passage d'un système symbolique inconscient à l'autre, préconscient/conscient, est donc corrélatif au passage d'un ensemble inconsistant, ouvert, à celui d'un système autre, fermé, donc consistant, mais qui, pour ce faire, doit présupposer cette relation d'extériorité de la chose au mot (comme représentation). Bref, d'un dehors par rapport à un système fermé. La particularité du psychisme, dans la théorie freudienne, c'est précisément sa constitution par le fonctionnement en parallèle de ces deux dimensions symboliques, ou, plus précisément, par ce fonctionnement selon un modèle topologique.

Ainsi, si, ordinairement ou intuitivement, nous croyons que le mot appelle toujours quelque chose d'externe pour pouvoir signifier (ce qui n'est autre chose que la base d'une théorie réaliste de la référence), du point de vue d'un système sui-référentiel, cette extériorité ne serait que l'ouverture même qui caractérise les associations d'objet.

Les représentations-mot se différencient alors des associations d'objet d'un point de vue presque uniquement fonctionnel. Ce qui change est alors la nature de la chose (externe) qui sert comme limite au système. Or, si le propre de l'appareil du langage, ainsi constitué, est d'avoir subsumé la chose sous la forme de l'objet - c'est-à-dire des associations qui sont déjà des traits symboliques -, le seul élément déclinant une nature autre que les éléments matériels du langage est l'inconsistance symbolique elle-même. Autrement dit, dans l'appareil psychique, c'est l'ouverture du système qui vient à la place du référent.

Ainsi, dès que nous passons du registre des représentations-mot à celui des associations d'objet, nous passons d'un registre qui se définit dans son opposition à quelque chose d'autre qui n'est pas lui - à un autre registre qui ne s'oppose plus à rien, et où l'extériorité doit être intérieure à lui-même. Ce qui nous soustrayions, enfin, c'est la logique même de la représentation (que conditionne la perception symbolique à se déployer sur un élément extérieur à son ensemble ou constitué d'une autre nature).

\section{Conclusion}

Comme nous avons essayé d'en démontrer, la théorie lacanienne, malgré l'utilisation de théories et de concepts étrangers aux références freudiennes originaux, ne se réduit nullement à la simple importation et application à la psychanalyse des concepts provenant de la linguistique structurale. Nous avons cherché dans le texte freudien, à partir des indications lacaniennes, les éléments théoriques qui se sont prêtés à l'interprétation structuraliste. Notre but était donc double : expliciter les concepts le plus saillants de l'interprétation lacanienne de l'inconscient en montrant que il s'agissait d'une interprétation rendu possible par l'œuvre de Freud elle-même, ainsi que, par le même mouvement, signaler la valeur heuristique de la démarche de Lacan, dans la mesure où cette démarche contribuait à rendre plus 
intelligible certains passages de la théorie freudienne de la représentation.

Néanmoins, nous ne considérons pas que l'idée de l'inconscient comme simplement structuré symboliquement soit l'alfa et l'oméga des théorisations lacaniennes à propos des rapports entre l'inconscient et le langage. Autour des années 1970, Lacan cherchera spécifier le mode d'adéquation entre son idée générale d'un «inconscient structuré comme un langage» et les considérations freudiennes à propos des jeux des mots présentés par l'association libre. L'exemple majeur de cette reformulation est illustré par Lacan (1973) dans son texte L'Étourdit. A partir de ce moment, l'inconscient n'est plus simplement une chaîne signifiante, définition uniquement formelle, mais l'intégrale d'équivoques qu'une langue concrète elle-même rend possible. Il s'agit alors de l'ensemble d'équivocités et d'ambigüités qui dépendent d'une langue maternelle en particulier, dont le concept de Lalangue sténographe l'existence. Selon Lacan :

"Ce dire [d'une énonciation quelconque] ne procède que du fait que l'inconscient, d'être structuré comme un langage, c'est-à-dire lalangue qu'il habite, est assujetti à l'équivoque dont chacune se distingue. Une langue entre autres n'est rien de plus que l'intégrale des équivoques que son histoire y a laissé persister». (Lacan, 1973, p. 47).

Notre article ne prétend pas s'occuper de cette reformulation assez tardive de la théorie lacanienne, mais uniquement du moment dans lequel Lacan propose la relecture structurelle de Freud. Cependant, nous considérons qu'il s'agit d'un moment d'importance majeure dans l'enseignement lacanien dont il faut marquer ainsi l'occurrence.

\section{Références}

Althusser, L. (1973). Lire le Capital (tome I). Paris: Maspero.

Freud, S. (1983). Contribution à la conception des aphasies. Paris: P.U.F. (originel publié en 1891).

Freud, S. (1895). Esquisse d'une psychologie scientifique. In S. Freud (Ed.), La naissance de la psychanalyse (1969, pp. Xxx). Paris: P.U.F.
Freud, S. (1896a). Lettre 46. In S. Freud (Ed.), La naissance de la psychanalyse (1969, pp. 143-148). Paris: P.U.F.

Freud, S. (1896b). Lettre 52. In S. Freud, (Ed.), La naissance de la psychanalyse (1969, pp. 153-160). Paris: P.U.F.

Freud, S. (2004). L'interprétation des rêves. In Euvres Complètes - Vol. IV. Paris: P.U.F. (Originel publié en 1900).

Koyré, A. (1947). Épiménide - le menteur. Paris: Hermann et compagnie.

Lacan, J. (1953). Fonction et champ de la parole et du langage. In J. Lacan (1966), Écrits (pp. 237-322). Paris: Éditions du Seuil.

Lacan, J. (1955). La chose freudienne ou sens du retour à Freud en psychanalyse. In J. Lacan (1966), Écrits (pp. 401-436). Paris: Éditions du Seuil.

Lacan, J. (1955/1956). Le Séminaire, Livre III: Les Psychoses. Paris: AFI. s/d.

Lacan, J. (1956). Compte rendu du « Discours de Rome et réponses aux interventions ». In Revue La Psychanalyse, 1, 202-211, et 242-255.

Lacan, J. (1957). L'instance de la lettre dans l'inconscient ou la raison depuis Freud. In J. Lacan (1966), Écrits (pp. 493-530). Paris: Éditions du Seuil.

Lacan, J. (1958). D'une question préliminaire à tout traitement possible de la psychose. In J. Lacan (1966), Écrits (pp. 531584). Paris: Éditions du Seuil.

Lacan, J. (1959/1960). Le Séminaire, livre VII: L'Éthique de la psychanalyse. Paris: AFI. s/d.

Lacan, J. (1964/1965). Le Séminaire, livre XI: Les Quatre concepts fondamentaux de la psychanalyse. Paris: AFI. s/d.

Lacan, J. (1973). L'Etourdit. En Scilicet (pp. 5-52). Paris: Seuil (Originel publié en 1972).

Russell, B. (1989). Écrits de logique philosophique. Paris: P.U.F.

Soler, C. (2004). Ce que Lacan disait des femmes. Paris : Éditions du Champ Lacanien. 